EuropeCalling

\title{
New immunisation recommendations in Germany
}

$\mathrm{T}$ here are recommendations for immunisations in virtually all European countries and in most countries in the world. They have been established by advisory boards in individual countries and comprise immunisations against various different target diseases with different schedules and vaccines depending on availability in each country. With respect to pertussis, for example, almost every European country has a different schedule and each is probably based on "good reasons".

In July 2001, updated recommendations were published for Germany (see www.rki.de/GESUND/IMPFEN/STIKO/ STIKO.HTM), They are the result of regular consultations among vaccine experts in the fields of paediatrics, internal medicine, public health, occupational medicine, microbiology, and the German Ministry of Health associated Robert Koch-Institute.

\section{UNCHANGED \\ RECOMMENDATIONS}

What is new and in which respect are they different from other European countries? Immunisations against 9 diseases-diphtheria, tetanus, pertussis (acellular), poliomyelitis (inactivated vaccine only), invasive $\mathrm{HiB}$ infection, hepatitis B, measles, mumps, and rubella-have been generally recommended for several years and this list has not become longer. The primary series of $\mathrm{DTPa} / \mathrm{HiB} / \mathrm{IPV} / \mathrm{HepB}$ comprises 4 doses at 2,3,4, and 11-14 months of age if given in combination. Hexavalent combination vaccines are available. A fifth dose of DT is recommended at 4 to 5 years of age, whereas a booster dose against poliomyelitis is recommended at any time between 9 and 17 years of age. In 2000, a fifth dose against pertussis was also introduced for 9 to 17 year old children and adolescents. This made Germany the first European country to introduce a routine booster dose against pertussis for adolescents, reacting to numerous reports showing the disease as a significant cause of persistent cough in this age group. France has now adopted the same policy. It is not difficult to predict that other countries will also adopt this strategy.
Immunisation against hepatitis B is not only recommended for all infants in Germany, but also as a 3 dose schedule for previously unimmunised 11 to 15 year olds.

\section{WHAT IS NEW?}

Earlier age for second dose of MMR The first dose of MMR is given in Germany from 11 months of age on. A second dose-known to be essential to close immunisation gaps due to primary vaccine failure-was implemented in 1991 to be given at 4-7 years of age. This recommendation was based on the practical grounds of easily scheduling MMR together with the pre-school DT booster. However, neither the first dose (coverage approximately $80-85 \%$ ) nor the second dose $(15 \%)$ have been taken up adequately, the main reason being the belief, still held by some, that measles is harmless and that it is "good" for a child to have natural disease. As a consequence, measles (and mumps and rubella for that matter) virus still circulates in the population and children with primary vaccine failure run a relatively high risk of acquiring the disease before they reach the age for the recommended second dose. Therefore, the second dose of MMR is now recommended soon after the first dose-that is, at 15 to 23 months of age. Switzerland, with similar problems of MMR acceptance, is in the process of implementing this change, too. It is hoped that the close sequence of MMR- 1 and MMR-2 will increase coverage rates, which would be an important step towards the goal of eliminating measles, rubella embryopathies, and also to reduce the burden of mumps.

\section{New recommendations for} individuals with increased risk of disease: pneumococcal, varicella, and pertussis vaccination

No European country has yet followed the US example of generally recommending pneumococcal conjugate vaccine for infants. This hesitation has various reasons, including —at least in some countries-the lack of epidemiological data to justify a general recommendation, anticipated difficulties with acceptance by parents ("one more shot for my baby, doctor?") and the significant costs of the programme relative to other childhood vaccines. German doctors are now recommended to administer a series of up to 4 immunisations with pneumococcal conjugate vaccine for children younger than 2 years of age with immunodeficiency, certain other chronic diseases, and for infants less than 38 weeks gestation. Although this will undoubtedly be a major benefit for affected individuals, the impact on the overall burden of invasive pneumococcal disease in children will probably be small.

Immunisation against varicella zoster virus (VZV) infections has been recommended for certain high risk groups in Germany for many years. They are defined as seronegative patients (1) scheduled for immunosuppressive therapy, (2) with leukaemia in remission, (3) with severe atopic eczema, (4) females who intend to become pregnant, and (5) health care workers. Now, a further target group has been defined for VZV immunisation: adolescents 12 to 15 years of age with no history of chickenpox and without previous VZV immunisation or serological demonstration of immunity. Although this group will be rather small (approximately 10\% of all adolescents, half of whom are false negatives), the implication of this new recommendation may be considerable: fewer primary VZV infections in adolescents and adults with a rather high complication rate and fewer cases of VZV embryopathies, which occur in Germany every year. The reliability of a positive history of chickenpox is very high, thus allowing this unique strategy without preceding individual serological screening.

Finally, indications for immunising adults against pertussis have been formulated. Based on several recent studies and epidemiological observations, a single dose of acellular pertussis vaccine is now recommended for health care workers working in divisions for infectious diseases or with children in general. Furthermore, personnel in kindergartens should be immunised. In contrast, the evidence for the need of pertussis immunisation for adults in general is still considered to be insufficient.

How these new recommendations will be taken by physicians in private practice (who administer most immunisations in Germany) and the public remains to be seen.

Ulrich Heininger European Editor 